\title{
A phenomenological model for improving understanding of the ammonium nitrate agglomeration process
}

\author{
Alvaro Videla Leiva $^{1, a}$, and Catalina Polanco Palacios ${ }^{1}$ \\ ${ }^{1}$ Mining Engineering Department, Pontificia Universidad Catolica de Chile, Chile
}

\begin{abstract}
Ammonium nitrate is intensively used as explosive in the mining industry as the main component of ANFO. The ammonium nitrate is known to be a strong hygroscopic crystal matter which generates problems due to the creation of water bridges between crystals leading later to nucleation and crystallization forming an agglomerated solid cake. The agglomeration process damages the ammonium nitrate performance and is undesirable. Usually either organic or inorganic coatings are used to control agglomeration. In the present work a characterization method of humidity adsorption of the ammonium nitrate crystal was performed under laboratory conditions. Several samples were exposed into a defined humidity in a controlled chamber during 5 hours after which the samples were tested to measure agglomeration as the resistance force to compression. A clear relation was found between coating protection level, humidity and agglomeration. Agglomeration can be then predicted by a phenomenological model based of combination of the mono-layer BET adsorption and CNT nucleation models.
\end{abstract}

${ }^{\text {a }}$ Corresponding author: avidela@ing.puc.cl 